\section{Technikakzeptanz und Technikteilhabe}

\author{
von Eva-Maria Jakobs, RWTH Aachen
}

Der Beitrag diskutiert Technikakzeptanz und -teilhabe von Gymnasiasten und Technikstudierenden als Nutzer von Technik und Techniknachwuchs. Basis ist eine aktuelle Studie zu Technikkonzepten, -nutzung und -bewertung dieser Zielgruppen und ihrer Einstellung zu technischen Berufen. Die Technikakzeptanz ist bei Schülern hoch, aber folgenlos. Sie bewirkt weder Formen der Auseinandersetzung mit Technik noch den Wunsch, einen technischen Beruf zu ergreifen. Ursachen werden in der Technikentwicklung selbst, in der Vermittlung technischen Wissens und dem Image technischer Studienfächer, des Ingenieurberufs und der Forschung gesehen. Lösungsansätze dafür könnten die Einführung eines eigenständigen Faches "Technik“, interdisziplinärer nutzerorientierter Technikforschung und -gestaltung, die Gestaltung technischer Studienfächer und die Korrektur von Stereotypen liefern.

\section{Leitfragen}

Es gibt viele Studien, die sich mit der Einstellung Deutscher zu Technik befassen. Der Akzeptanzbegriff bleibt v. a. in Umfragestudien häufig vage. Meint Akzeptanz wohl erwogene positive Annahme, ungeprüftes, unreflektiertes Befürworten oder Duldung? Geht es um Einstellungen oder Wertsysteme? Hat Akzeptanz Konsequenzen? Löst sie Handlungsdispositionen aus wie Kauflust oder die Bereitschaft, einen technischen Beruf $\mathrm{zu}$ ergreifen? Richtet sich die Akzeptanzdiskussion auf Technik im Sinne technischer Artefakte oder auf Technologien im Sinne der dahinter stehenden Prinzipien und Wissensbestände? Gibt es eine alles umgreifende allgemeine Akzeptanz oder differieren Urteile bereichs- und objektbezogen nach Zugehörigkeit zu sozialen oder kulturellen (Alters-)Gruppen? Wenn letzteres zutrifft, wovon ist die Rede, wenn Politik und Wirtschaft Akzeptanz für Innovationen, Technik und den Industriestandort Deutschland fordern? Welche Interessen verbinden sich damit? Mit anderen Worten: Wofür benötigt Technik Akzeptanz bzw. eine Gesellschaft Technikakzeptanz? Mit diesen Fragen haben sich verschiedene Disziplinen befasst, sie sind weder neu (vgl. etwa Jaufmann, Kistler 1988) noch abschließend zu beantworten.

Dass man sie immer wieder stellen sollte, zeigen Diskussionen in den Kreisen der Betroffenen - z. B. bei Ingenieuren in Forschung, Lehre und Praxis, die den ausbleibenden Zustrom technischen Nachwuchses verkürzend auf latente Technikfeindlichkeit und Desinteresse zurückführen. Sie verstellen sich damit den Blick auf andere Gründe und daran gebundene Handlungsoptionen. Bemerkenswert ist die mentale Ausblendung von Umfrageergebnissen, die seit Ende des 20. Jahrhunderts gegen die Annahme von Technikfeindlichkeit sprechen.

Der vorliegende Beitrag diskutiert spezielle Aspekte von Technikakzeptanz. Er fokussiert junge Erwachsene in ihrer Eigenschaft als Nutzer von Technik und potenzieller Nachwuchs für die Technikentwicklung von morgen. Er fragt nach dem Zusammenhang unterschiedlicher

- Technikeigenschaften und Akzeptanz: Für welchen Typ von Technik ist Akzeptanz notwendig, erwart- oder erzeugbar? Was interessiert bzw. stört Nutzer an Technik und wie beeinflusst dies die Wahrnehmung und Bewertung von Technik?

- Technikakzeptanz und Wissen: Wie ,begreifbar" sind moderne Technologien? Wie viel Wissen benötigt der Nutzer, um Technik nutzen und/oder angemessen bewerten zu können?

- Technikakzeptanz und Altersgruppe: Wie sieht das technische Selbstverständnis von Altergruppen aus?

- Technikakzeptanz und Teilhabe: Reicht Akzeptanz für den Wunsch nach aktiver Teilhabe? Welche Rahmenbedingungen motivieren junge Menschen, durch die Wahl eines technischen Berufes zur Gestaltung von Technik beizutragen?

Der letztgenannte Zusammenhang ist für technische Hochschulen von besonderem Interesse. Die Adressierung technischen Nachwuchses bedingt eine möglichst genaue Kenntnis der Wünsche, Ziele und Einstellungen junger Menschen (zu Technik). 


\section{Technikkonzepte, -nutzung und -bewertung junger Erwachsener}

Es gibt verschiedene aktuelle Studien, die sich mit der Sicht von Jugendlichen auf Technik befassen, so z. B. die Shell-Jugendstudie (Deutsche Shell 2004), die „Generation-05“Studie des „manager magazin“ (2004) und die Studie „Technophil oder technophob? Eine Studie zur altersspezifischen Konzeptualisierung von Technik“" (Jakobs et al. 2005) ${ }^{1}$. Die erstgenannten Studien fokussieren Studierende, die letztgenannte adressiert Gymnasiasten (Klassenstufe 11, 12) und Technikstudierende (im Hauptstudium). In der Studie „Technophil oder technophob?" wurden junge Erwachsene $\mathrm{zu}$ Themen wie Techniknutzung und bewertung, Lebens- und Berufsvorstellung sowie ihre Einstellung zu technischen Fächern und Forschung befragt. Hinter der Studie steht das Interesse technischer Hochschulen, Hinweise auf Rahmenbedingungen und Faktoren zu erhalten, die junge Menschen für Technik und ein technisches Studium interessieren, um sie gezielter adressieren und für ein solches Studium gewinnen zu können. In einer Pilotstudie wurden Vertreter der Zielgruppen in Doppelinterviews und Fokusgruppen befragt und die Ergebnisse anschließend per Fragebogen quantitativ geprüft. An der Studie beteiligten sich 540 Schüler mono- und koedukativer Gymnasien sowie 550 Studierende. Die Ergebnisse der Studie decken sich in vielen Punkten mit denen anderer Erhebungen und bieten Ansatzpunkte für die folgende Akzeptanzdiskussion. Der Fokus gilt hier der Zielgruppe Gymnasiasten.

\subsection{Akzeptanz für Technik oder Technologie?}

In der öffentlichen Diskussion wird in der Regel der Ausdruck Technikakzeptanz verwendet. Die befragten Gymnasiasten unterscheiden dagegen Technik und Technologie. Technik gilt als Umsetzung von Technologien und als solche als sehr real und konkret. Technologie wird dagegen als abstrakte, gedankliche Leistung verstanden, als Gegenstand und Ergebnis wissenschaftlicher Forschung, die sich der Wahrnehmung der Schüler entzieht.
„Technologie ist für mich die Entwicklung von irgendwelchen Sachen, und Technik ist das, was wir von der Technologie - also von dieser Wissenschaft - haben in unserem Haushalt.“ [Schüler, 18 Jahre]

Die Befragten bewerten Technik und technologische Innovation als wichtig für Deutschland und für ihre Zukunft. Ihre Sicht variiert abhängig von ihrem (lebensweltlichen) Bezug wie auch bereichs- und objektabhängig. Der Bezug zu Alltagstechnik ist ein anderer als zu technologischer Forschung und Großtechnologie.

Technik nehmen sie in erster Linie als Alltagstechnik wahr; Alltagstechnik ist für sie primär Informations- und Kommunikationstechnik (IuK); andere technische Produkte (z. B. Transport- oder Haushaltstechnik) scheinen nicht erwähnenswert. Als wichtigste technische Errungenschaft der letzten zwanzig Jahre gelten Mobiltelefon, Internet und Computer. Artefakte dieser Art sind unverzichtbare Hilfsmittel ihres Alltags und bestimmen ihr generationsspezifisches Bild von Technik (ähnlich Pfennig et al. 2002).

Die Aussagen zu Technologien deuten auf eine tendenziell positive Bewertung: Die Mehrheit ist der Meinung, technologische Entwicklung bedeutet Fortschritt und hilft, Alltagsprobleme zu lösen. Abhängig von dem mit der Technologie zu lösenden Problem und/oder den Konsequenzen ihrer Anwendung fällt das Urteil positiv, distanziert oder ablehnend aus:

\section{„Es gibt Technologien, die dem Menschen helfen, den Menschen unterstützen. Es gibt welche, die den Menschen faul machen. Und es gibt Technologien, die retten Leben. Die finde ich gut und die anderen, die finde ich halt unnötig, die kann man auf jeden Fall ab- schaffen." [Schüler, 18 Jahre] \\ „Also alles, was mit Massenvernichtungs- waffen zu tun hat, würde ich schon mal als schlecht abtun. Dann als gut, was die Um- welt schont und praktikabel und nicht zu teu- er ist." [Schüler, 19 Jahre]}

Die Mehrheit ist sich einig, dass vor allem Technologien gefördert werden sollten, die Arbeitsplätze schaffen. Zum Teil zeichnet sich die Sorge ab, dass Technikentwicklung Arbeitsplätze eher vernichtet als schafft und der Arbeitsmarkt für Ingenieure unsicher ist. 


\subsection{Techniknutzung und Akzeptanz}

Die meisten der Befragten bezeichnen sich als technisch versiert (im Umgang mit IuK). Begründet wird die Fähigkeit des souveränen Umgangs mit dem Generationenfaktor. Ohne bewusst an Technik herangeführt worden zu sein, habe sie der tägliche Umgang mit Technik und sich schnell verändernde Bedienanforderungen geschult. Ihre Generation besitze damit einen kaum zu überbietenden Vorteil gegenüber anderen, besonders älteren Menschen.

„(...), weil gerade unsere Generation damit aufgewachsen ist, haben wir (...) dieses logische Denken, mit Technik umzugehen.“ [Schüler, 18 Jahre]

,(...) unsere Eltern oder die Lehrer (...) sind ja aufgewachsen, wo es noch nicht alles so technisiert war. Wir sind da ja schon fast von Geburt an, wir sind da so reingewachsen und wenn man da irgendwie täglich Umgang mit hat, ist es wesentlich einfacher, damit umzugehen.“ [Schüler, 19 Jahre ]

Die Techniknutzung erfolgt pragmatisch, genauer zweck- und sachorientiert. Der Aufwand an Kosten und Zeit soll gering, der Nutzen groß sein.

\subsection{Technikeigenschaften und Akzeptanz}

Ein Interesse unserer Befragung war, Hinweise darauf zu erhalten, wie sich Technikeigenschaften auf die Technikbewertung auswirken: Wie müssen technische Produkte gestaltet sein, um aus der Sicht des Nutzers akzeptabel zu sein?

Das von den Schülern (postulierte) Interesse an Alltagstechnik erreicht seine Grenzen, wenn die genutzte Technik nicht wie gewünscht funktioniert. $\mathrm{Zu}$ den Merkmalen der befragten Gruppe gehört eine geringe bzw. fehlende Bereitschaft, sich mit der Funktionalität technischer Geräte, ihrer Beschreibung und auftretenden Problemen auseinanderzusetzen. Das Interesse ist passiv und konsumorientiert. Technik muss aus sich heraus funktionieren und verständlich sein, um positiv wahrgenommen zu werden:

„Mir macht das nur Spaß, wenn ich das sofort verstehe, wenn das dann so kompliziert ist, dass man sich da einlesen muss und so, ist natürlich toll, das dann nachher so zu können, aber ich habe da nicht so die Geduld, also ich lass mir das dann auch lieber von wem anders erklären.“ [Schülerin, 17 Jahre]

Die Forderung nach reibungslos funktionierender Technik, die sich in ihrem Funktionieren gleichsam dem Bewusstsein entzieht, scheint eine Forderung, die Nutzer aller Altersklassen verbindet. Ihr stehen in der Praxis oft defizitär gestaltete, d. h. nicht intuitiv erschließbare technische Produkte entgegen.

Der Informations- und Klärungsbedarf des Nutzers soll durch die technische Dokumentation gedeckt werden. Viele Dokumentationen erfüllen diese Aufgabe eher mangelhaft - die Nutzer reagieren irritiert. Eine im Auftrag der Zeitschrift „GEO“ angefertigte Studie, in der 2.000 Deutsche zu ihren Problemen mit Alltagstechnik befragt wurden, ergab unverständliche Gebrauchsanweisungen als häufigsten Kritikpunkt (73 Prozent) (Broschart 2005). Die Kombination mit anderen Mängeln - zu hohe Reparaturkosten, schnell veraltende Technik, Funktionsüberfluss, mangelhafter Service - erzeugt Unmut und Unlust, z. B. sich mit Technik zu befassen. Bezogen auf neue Technologieanwendungen wie z. B. „smarte Gegenstände“ ist unklar, welche Probleme an der Mensch/TechnikSchnittstelle erwartbar sind und wie sie vermieden werden können. Für Innovationen dieser Art sollte möglichst früh geprüft werden, welche Applikationen und Anwendungsszenarien den Nutzer erreichen. Dies setzt eine interdisziplinäre nutzerorientierte Technikforschung voraus.

Der Erwerb von Technik ist im Falle der von uns befragten Gymnasiasten von Kosten/ Nutzen-Erwägungen bestimmt. Weitere Motive ergeben sich durch soziale und technologische Zugzwänge.

„Vom Nutzen her würde ich sagen, ob es was bringt, ob ich es benutze im Alltag." [Schülerin, 18 Jahre]

„Wenn man (...) was Neues kauft, dann nur aufgrund der Notwendigkeit, weil es (...) einfach keine Filme mehr auf Video (gibt), da muss man sich früher oder später einen DVD-Player anschaffen." [Schüler, 17 Jahre]

„Das Handy habe ich irgendwann mal bekommen, weil ich es brauchte und weil halt jeder eins hatte, aber nicht nur deshalb, sondern weil man (...) unterwegs ist und die Eltern wissen wollen, wo man ist (...).“ [Schülerin, 18 Jahre] 
Spielerische Aspekte scheinen - im Gegensatz zu dem im öffentlichen Diskurs über Jugendliche und ihre IuK-Nutzung proklamierten Spieldrang - in dieser Phase ihrer Techniksozialisation nicht (mehr) wichtig.

\begin{abstract}
„Eigentlich nur für SMS und Anrufe, also diese Spiele darauf nutze ich eigentlich nicht wirklich." [Schülerin, 18 Jahre]

„Weil man da ja voll pubertär (war), alles haben musste, was die anderen hatten, aber auch, weil das so für die Kommunikation so wichtig war. Also, weil alle sich SMS geschrieben hatten und man hatte selber kein Handy und war dann so ein bisschen abgeschnitten von der Außenwelt." [Schülerin, 18 Jahre]
\end{abstract}

Hier wäre genauer zu klären, welche Phänomene der Techniknutzung (zeit- und kulturbezogen) an die Zugehörigkeit zu sozialen Gruppen, die Ausbildung und/oder Parameter wie Alter und Geschlecht gebunden sind. Ein anderer Aspekt betrifft Langzeitstudien zu Techniksozialisationen und der Wahrnehmung von Technik. Diese beschäftigen sich mit den Fragen: Wann und warum ändert sich die Sicht auf Technik und welche Konsequenzen hat dies für die Technikwahrnehmung und -akzeptanz?

\subsection{Akzeptanz: ja - Teilhabe: nein?}

Insgesamt zeigt sich eine breite Akzeptanz für Technik. Junge Erwachsene akzeptieren (Alltags-)Technik, diese Akzeptanz ist jedoch weitgehend ,folgenlos“: Sie führt weder zur Bereitschaft, sich mit Technik auseinanderzusetzen, noch zur Bereitschaft, sich zu informieren (nur Wenige lesen technische Artikel oder Magazine), noch zu einem Mehr an technischem Wissen. So kann z. B. kaum einer der Befragten erläutern, wie ein Mobiltelefon funktioniert. Nur ein geringer Prozentsatz (6,3 Prozent) interessiert sich für ein technisches Studium; kaum einer plant eine wissenschaftliche Karriere (vgl. Abschnitt 2.6).

Auch die Verantwortung für technische Entwicklungen und die hier geforderte Kontrolle und Steuerung wird an andere (eine mehr oder weniger anonyme Öffentlichkeit) delegiert. Insgesamt bestätigt sich der seit längerem beobachtbare Rückzug ins Private (ähnlich Pfennig et al. 2002; Deutsche Shell 2004; Generation-05-Studie 2005).

\subsection{Hemmschwellen für Technik- partizipation}

Die geringe Neigung zur Auseinandersetzung mit Technik legt die Frage nahe, woran dies liegt. Für den Befund gibt es verschiedene Erklärungsansätze:

Technik ist so allgegenwärtig und normal, dass sie nicht mehr bewusst wahrgenommen wird.

Technische Artefakte gehören zum Lebensalltag der von uns befragten jungen Erwachsenen, sie werden als inhärenter, selbstverständlicher Bestandteil wahrgenommen. Zumindest für den Bereich der IuK-Technik ist Pfennig, Renn und Mack (2002, S. 96) zu widersprechen, die in Schulen fehlende Auseinandersetzung mit Technik verhindere, dass Schüler Technik als Teil des Alltags wahrnehmen. Sie nehmen sie sehr wohl wahr. Was fehlt, ist die kritische Auseinandersetzung mit den (kulturellen, sozialen und kognitiven) Potenzialen und Kosten ihrer Nutzung.

\section{Die Vermittlung technischen Wissens und Könnens reicht nicht aus.}

Um mit neuen technischen Produkten umgehen und dabei auftretende Probleme meistern zu können, benötigt der Anwender ein bestimmtes technisches Wissen bzw. Verständnis. Es ermöglicht ihm den sachgerechten Umgang mit Technik, die Reflexion von Technik, die Partizipation an Technik sowie die Einschätzung potenzieller Wechselwirkungen von Technik, Gesellschaft und Kultur; kurz: Wissen ist eine wesentliche Grundvoraussetzung für „mündige“ Nutzer. Unklar ist jedoch, was dieses Wissen oder Verständnis ausmacht: Welches Wissen und Können braucht der Nutzer, um partizipieren (verstehen, mitgestalten, entscheiden) zu können? Wer soll es vermitteln und welche Konsequenzen hat es, wenn weite Teile der Bevölkerung nicht darüber verfügen?

Technisches Wissen wird ab Sekundarstufe II primär in den naturwissenschaftlichen Fächern vermittelt - v. a. im Fach Physik. Die Vermittlung konzentriert sich auf Technik als Anwendung naturwissenschaftlicher Prinzipien und Verfahren. Schüler, die sich für Technik interessieren, aber die hohen Anforderungen 
und Misserfolgschancen des Physikunterrichts scheuen, fallen aus dem Vermittlungsraster heraus. Umgekehrt führt das Interesse für Physik und Mathematik nicht automatisch zu technischem Interesse (vgl. Jakobs et al. 2005).

- Das für das Verständnis neuer Technologien notwendige Wissen ist an sich nicht vermittelbar.

- Technik (mit einem hohen Elektronikanteil) ist Laien (wie Experten) zunehmend unzugänglich.

Wenn das Technikkonzept des einzelnen von der Alltagstechnik bestimmt wird, mit der er aufwächst bzw. die ihn täglich umgibt, dann ist zu fragen, was geschieht, wenn diese Technik im wörtlichen Sinne „unbegreifbar“ wird. Früher war es möglich, als Kind den elterlichen Wecker zu demontieren und mehr oder weniger erfolgreich wieder zusammenzusetzen. Heute entzieht der Trend zu unsichtbarer Alltagstechnik (z. B. „Intelligent Ambiente“) dem Nutzer den Boden für die Auseinandersetzung mit ihr. Der Wegfall sinnlich wahrnehmbaren Begreifens von Technik erschwert den Zugang zu Technik. Die den Nutzer umgebende Technik wird ihm erst bewusst, wenn sie nicht oder fehlerhaft funktioniert. Angemessenes Reagieren setzt (in der Regel nicht vorhandenes) technisches Verständnis voraus. Nicht-Verstehen erzeugt Unsicherheit, Unsicherheit erzeugt Unwillen, Unwissen und Unwille erzeugen Feindbilder und/oder Ängste.

\subsection{Fehlende Attraktivität technischer Studien, Berufe und Forschung}

Die genannten Gründe mögen in der einen oder anderen Weise dazu beitragen, dass das Interesse an technischen Studienfächern gering ist. Dass dies nicht nur auf die Veränderung der Technik an sich oder die Ablösung der Bastlergeneration durch eine Anwendergeneration (Köcher 2004) zurückzuführen ist, sondern andere - vielleicht ergänzende, in jedem Fall jedoch ernst zu nehmende - Gründe hat, zeichnet sich in verschiedenen Studien ab. Die Gründe betreffen das eher schlechte, da unattraktive Image technischer Studien, Berufe und Forschung.

\section{Studienimage}

Die von uns befragten Gymnasiasten entscheiden sich im Wesentlichen aus zwei Gründen gegen technische Studienfächer: Sie gelten zum einen als anspruchsvoll, anstrengend, auf abstrakte, schwer verständliche Technologien gerichtet, zum anderen als sozial einseitig. In der Vorstellung der Schüler lässt das Studium kaum Raum für soziale Kontakte, Teamarbeit und Selbstverwirklichung, die ihnen jedoch wichtig sind. Das Interesse ist auch bei Schülern gering, die erfolgreich Leistungskurse in Mathematik und Physik belegen und sich für Technik interessieren.

Dass es sich nicht nur um Vorurteile handelt, zeigen die Aussagen der von uns befragten Studierenden der Technikwissenschaften. Viele sind unzufrieden mit ihrem Studium. Frustrationsgründe betreffen den Frontalunterricht, das Fehlen von Team- und Projektarbeit sowie einen geringen Praxisbezug. In ihrer Wahrnehmung spielen im Studium Teamarbeit und $\mathrm{Spa}$ kaum eine Rolle. Dasselbe gilt für Softskills wie z. B. sprachliche Ausdrucksfähigkeiten. Wintermantel et al. (2002) belegen andererseits anhand der Analyse von Stellenausschreibungen, dass der Stellenwert von Team- und Kommunikationsfähigkeit seit 1999 in Vergleich zu anderen Eigenschaften (wie z. B. Führungsqualitäten) ständig zunimmt.

\section{Berufsimage}

Auch der Ingenieurberuf hat geringe Anziehungskraft. Es gibt eine zunehmende ,innere Distanz", deren Ursachen wenig untersucht sind (vgl. aber Pfennig et al. 2002). ${ }^{2}$ Diesen Imageverlust führen Wintermantel et al. (2002) weniger auf Entwicklungen des Arbeitsmarktes zurück, die in den 1990er Jahren des zwanzigsten Jahrhunderts bestimmend waren, ${ }^{3}$ als auf eine sozial bedingte negative Stereotypenbildung und damit einhergehende Stigmatisierung des Ingenieurs:

- Das Ingenieur-Bild ist konservativ: Ingenieure gelten als fleißig, intelligent, kreativ, verantwortungsbewusst und erfolgreich (Wintermantel et al. 2002).

- Ingenieure gelten aber auch als ,introvertierte“ Tüftler. Ihnen werden im Vergleich 
$\mathrm{zu}$ anderen Berufsgruppen (z. B. Betriebswirte) sozial-kooperative Fähigkeiten (kollegiale Unterstützung am Arbeitsplatz, Teamgeist) und kommunikative Qualitäten eher ab- als zugeschrieben (dies. 2002; Jakobs et al. 2005). ${ }^{4}$

- Der Ingenieurberuf gilt nach wie vor als „männlich“ sowie als unvereinbar mit Familie (Wintermantel et al. 2002; Jakobs et al. 2005).

Das skizzierte Bild ist schwer vereinbar mit den Motiven, die die Berufs- und Lebensplanung junger Menschen bestimmen. Sie erfolgt bewusst und zielgerichtet. Wichtig sind Männern wie Frauen ein sicherer Arbeitsplatz, die Vereinbarkeit von Beruf und Familie sowie die Pflege von Freundschaften durch Kontinuität und Präsenz vor Ort. Der Wunsch nach Kindern wie auch nach Zeit für die Familie ist bei beiden Geschlechtern stark ausgeprägt (ähnlich: Deutsche Shell 2004, Generation-05-Studie 2005). Die befragten jungen Erwachsenen suchen private und berufliche Erfüllung. Traditionelle Motive wie Einkommen und Aufstiegschancen werden zunehmend durch Werte abgelöst wie Vielseitigkeit (Interessantheit) der Arbeit und soziale Interaktion. ${ }^{5}$ Der Trend gilt für Schüler wie Studierende (ähnlich Deutsche Shell 2004; Generation-05-Studie 2005). In diese Welt passt Technik, aber nicht der ,introvertierte Tüftler“.

Die von uns befragten Schüler befürchten unsichere Berufschancen aufgrund technischer Fortschritte. Ihre Einschätzung unterscheidet sich deutlich von der der Technikstudierenden. Letztere gehen von einer allgemeinen Wertschätzung ihres Berufes in der Bevölkerung aus, die sie mit dem Schwierigkeitsgrad ihrer Gegenstände und Ausbildung begründen:

„Ich denke, die haben immer noch eine Menge Respekt, weil (...) viele Leute sich halt nicht so mit Technik auskennen. Aber wenn die hören, oh ein Maschinenbauingenieur, dann wird erst mal gesagt, oh der ist bestimmt, der hat was auf dem Kasten. Viele trauen sich dann auch nicht so richtig dran oder fragen auch gar nicht groß nach, weil das noch so, ja schwierig ist, für Leute da rein zukommen." [Maschinenbaustudent, 21 Jahre]

Die Studierenden sind sich sicher, gute Chancen auf dem Arbeitsmarkt zu haben. Begründet werden die Aussichten mit dem guten Ruf ihrer Hochschule und der im Studium erworbenen
Qualifikation (im Fach Maschinenbau von 97, im Fach Elektrotechnik von 94 und im Fach Bauingenieurwesen von 83 Prozent der Befragten). Ganz anders dagegen die Einschätzung der in der Generation-05-Studie Befragten: Die Mehrzahl sieht ihre berufliche Zukunft in Deutschland gefährdet. In diesem Zusammenhang wäre zu diskutieren, wie sich das Image der ausbildenden Hochschule auf Befragungsergebnisse auswirkt.

\section{Forschung}

Die wenigsten der von uns befragten Schüler und Studierenden wollen in die Forschung gehen. Forschung genießt insgesamt ein schlechtes Image. Die wenigsten können sich etwas darunter vorstellen (zu Stereotypenbildung s. Hötticke 2001). Das Bild der Schüler wird durch Spielfilme und Romane geprägt (vgl. Weingart 2003). Forscher gelten als weltfremd bzw. schwer einschätzbar und daher als kontrollbedürftig.

\section{Fazit}

Bezogen auf die Forderung nach Akzeptanz für Innovationen wäre genauer $\mathrm{zu}$ hinterfragen, was damit gemeint ist. Wenn es um neutrale Offenheit geht, ist der Handlungsbedarf eher gering: Deutsche schätzen Technik - zumindest aus der Ferne. Wenn die Akzeptanzforderung dagegen der Technikentwicklung und der Bereitschaft, sich daran zu beteiligen, gilt, besteht erheblicher Handlungsbedarf. Die im Folgenden genannten Lösungsansätze sind zum Teil nicht neu, angesichts ihrer fehlenden Umsetzung jedoch weiter aktuell:

\section{Akzeptanz durch interdisziplinäre nutzerorien- tierte Technikforschung und -gestaltung}

Bezogen auf den Anwender von Technik ist die Chance auf Technikakzeptanz hoch, wenn technische Produkte seinen Interessen entgegenkommen, d. h. wenn sie ihn bei der Erfüllung seiner Interessen unterstützen. Um sinnvolle Kompromisse zwischen dem technisch Möglichem und dem menschlich Gewolltem zu erreichen, ist es sinnvoll und notwendig, den Nutzer in die Entwicklung technischer Produk- 
te einzubeziehen. Nutzerorientierte Technikgestaltung erfordert nutzerorientierte Technikforschung, die nur in der Zusammenarbeit von Vertretern verschiedener Disziplinen zu leisten ist. In diesem Kontext können die Human-, Sozial- und Geisteswissenschaften wertvolle Beiträge leisten.

\section{Technisches Verständnis durch Technikunter- richt in allen Schulformen und Klassenstufen}

Wer mündige Nutzer und technischen Nachwuchs will, muss in die Schulbildung investieren. Die Vermittlung technikbezogenen Wissens erfolgt insgesamt zu heterogen - je nach Bundesland, Schulform und schulischer Schwerpunktsetzung. Für die systematische Auseinandersetzung mit Technik über die Zeit hinweg wären eine bundesweite Einigung auf ein eigenständiges Fach „Technik" hilfreich, das bis zur Sekundarstufe II angeboten wird, sowie mehr spezielle Fördermöglichkeiten für technische Talente. Dazu gehören z. B. Technikgymnasien (ähnlich Pfennig et al. 2002).

Das Fach sollte Schülern den Facettenreichtum technischer Entwicklungen begreiflich und nachvollziehbar machen. Dazu gehören historische, soziale und kulturelle Aspekte von Alltags- und Großtechnologien genauso wie die Auseinandersetzung mit zweckrationalen und emotionalen Gestaltungselementen oder dem eigenen Tun. Mündige Nutzer sind Nutzer, die über technisches Wissen bzw. Verständnis verfügen und bereit sind, dieses zu nutzen. Auf der anderen Seite ist zu fragen, wie die Forderung nach einer attraktiven didaktischen Vermittlung von der pädagogischen Forschung und der Lehrerausbildung wahrgenommen und unterstützt wird. Hier sind $u$. a. auch die Ingenieurwissenschaften selbst gefordert. Wer Nachwuchs will, muss auch bereit sein, in die Berufsgruppe zu investieren, die den Nachwuchs heranbildet.

\section{Nachwuchsgewinnung durch attraktive Studieninhalte und Arbeitsbedingungen}

Potenziale in der Ingenieurausbildung betreffen Vermittlungsformen (weniger Frontalunterricht, mehr Interaktion zwischen Lehrenden und Lernenden) und -inhalte (mehr Raum für den Erwerb berufsrelevanter sozialer Fähigkeiten, wie Team- und Kommunikationsfähigkeit) sowie den Praxisbezug (Veranstaltungen, die früh im Studium den Bezug zu den eigentlichen Gegenständen der gewählten Technikdisziplin herstellen, konkrete Beispiele und Projektarbeit). Wünschenswert wäre die Integration der Nutzerperspektive in das ingenieurwissenschaftliche Studium wie auch eine Ausbildung, die das Bewusstsein für den Gewinn der Blickerweiterung und der Zusammenarbeit mit anderen Disziplinen schärft. Beide Forderungen erlauben zusätzliche Anreize für die Aufnahme eines ingenieurwissenschaftlichen Studiums.

Bezogen auf die Berufspraxis sind Maßnahmen erforderlich, die Familie und Beruf für Mann und Frau vereinbar machen, z. B. Formen der betriebseigenen Kinderbetreuung. Handlungsbedarf betrifft aber auch die Korrektur von Berufs- und Wissenschaftsstereotypen. Wenn Film und Fernsehen maßgeblich Vorstellungen prägen, wäre darüber nachzudenken, wie diese Möglichkeiten sinnvoll genutzt werden können - z. B. durch eine Soap, in der Mensch und Technik in ihrem facettenreichen Zusammenspiel eine Hauptrolle erhalten.

\section{Anmerkungen}

1) Finanziert wurde diese Studie durch die WalterEversheim-Stiftung, den VDE, Siemens-CKI Aachen und die Otto-Junker-Stiftung. Eine zweite Studie zum Umgang älterer Menschen mit Technik und ihre Sicht auf Technik wird Anfang 2006 vorliegen.

2) Auf die Gruppe der 16- bis 19Jährigen trifft die Aussage, jeder fünfte Deutsche zähle diesen Beruf zu den wichtigsten (Köcher 2004), nicht zu.

3) Dazu gehören z. B. Arbeitsmarktpolitik und Konjunkturschwankungen (vgl. Pfennig et al. 2002).

4) Die stereotype Zuschreibung geht gänzlich an der Praxis vorbei: Die Arbeit von Ingenieuren ist stark teamorientiert, Kommunikation spielt eine wichtige Rolle in ihrem Berufsleben z. B. bei der Koordinierung von Arbeit (vgl. Jakobs 2005).

5) Der Befund wird je nach Ansatz unterschiedlich eingeschätzt. Einige sprechen von einer Hinwendung zu liberal-individualistischen Werten, andere vom Erstarken post-materialistischer Werte, die in Nachindustriegesellschaften neben Wertkriterien der Leistung treten. Auswirkungen auf Lernverhalten und Lernerfolge untersucht das Teilprojekt „Gesellschaftlicher Wertewandel und seine Konsequenzen für die allgemeine schulische und mathematisch-naturwissenschaftliche 
Lernmotivation“ des DFG-Schwerpunktprogramms BIQUA an der Universität Mannheim.

\section{Literatur}

Broschart, J., 2005: Technik im Alltag: Freund oder Feind? In: Geo 5/2005, S. 48-74

Deutsche Shell (Hrsg.), 2004: Jugend 2002. Zwischen pragmatischen Idealismus und robustem Materialismus (14. Shell Jugendstudie). Frankfurt a. M.: Fischer

Generation-05-Studie, 2004: http://www.managermagazin.de/koepfe/karriere/0,2828,346667,00.html

Hötticke, D., 2001: Die Vorstellungen von Schülern und Schülerinnen von der „Natur der Naturwissenschaften“. In: Zeitschrift für Didaktik der Naturwissenschaften 7 (2005), S. 7-23

Jakobs, E.-M., 2005: Writing at work. In: Jakobs, E.-M.; Lehnen, K., Schindler, K. (Hrsg.): Schreiben am Arbeitsplatz. Wiesbaden: Verlag für Sozialwissenschaften, S. 13-42

Jakobs, E.-M.; Schindler, K.; Straetmans, S., 2005: Technophil oder technophob? Eine Studie zur altersabhängigen Konzeptualisierung von Technik. Aachen (beziehbar über die Walter-Eversheim-Stiftung c/o FIR, Pontdriesch 14-16, D-52062 Aachen)

Jaufmann, D.; Kistler, E., (Hrsg.), 1988: Sind die Deutschen technikfeindlich? Erkenntnis oder Vorurteil. Opladen: Leske+Budrich

Köcher, R., 2004: Technikfeindlich und innovationsmüde? In: Dokumentation des AcatechSymposium „Innovationsfähigkeit“”. 11. Mai 2004. Acatech e.V., München, S. 34-37

Pfennig, U.; Renn, O.; Mack, U., 2002: Zur Zukunft technischer und naturwissenschaftlicher Berufe. Strategien gegen den Nachwuchsmangel. Stuttgart: Akademie für Technikfolgenabschätzung in BadenWürttemberg

Weingart, P., 2003: Von Menschenzüchtern, Weltbeherrschern und skrupellosen Genies - Das Bild der Wissenschaft im Spielfilm. In: Iglhaut, S., Spring, T. (Hrsg.): Zwischen Nanowelt und globaler Kultur. Science Fiction, Bilder und Texte. Berlin: Jovis Verlag, S. 211-228

Wintermantel, M.; Plach, M.; Behmann, H.; Scheck, I., 2002: Entwicklungs- und sozialpsychologische Determinanten der Entscheidung für ein ingenieuroder naturwissenschaftliches Studium. 2. Zwischenbericht. Universität des Saarlandes. Lehrstuhl für Sozialpsychologie

\section{Kontakt}

Prof. Dr. Eva-Maria Jakobs

Institut für Sprach- und Kommunikationswissenschaft

Textlinguistik/Technikkommunikation RWTH Aachen

Templergraben 83, 52062 Aachen

Tel.: +49 (0) 241 - 8093563

E-Mail: e.m.jakobs@tk.rwth-aachen.de

Internet: http://www.tl.rwth-aachen.de 